\title{
Characterization of polyvinyl alcohol/acrylamide holographic memories with a first-harmonic diffusion model
}

\author{
Sergi Gallego, Manuel Ortuño, Cristian Neipp, Andrés Márquez, Augusto Beléndez, \\ and Inmaculada Pascual
}

\begin{abstract}
Several theoretical models have been proposed to predict the behavior of photopolymers as holographic recording materials. Basically these models have been applied to study thin layers (around $100 \mu \mathrm{m}$ thick). The increasing importance of holographic memories recorded in photopolymers (thickness of $>500 \mu \mathrm{m}$ ) makes it necessary to extend the ideas proposed by these models to study thick photopolymer layers. We calculate the temporal evolution of the diffraction efficiencies for thick layers using a firstharmonic diffusion model, and the results obtained are compared with the corresponding values for thin layers. Furthermore, the values of the average diffusivity of the polymer chains after the grating is formed are also obtained. In general, we find that the monomer and polymer diffusivity increases when higher values of thickness are used. (C) 2005 Optical Society of America
\end{abstract}

OCIS codes: $\quad 090.0090,090.2900,090.7330$.

\section{Introduction}

In recent years, research to achieve optimized holographic memories has received much attention, ${ }^{1-4}$ and new companies have optimized thick photopolymers for data storage. ${ }^{5}$ This type of device takes advantage of the thickness of the recording material to record data, a characteristic that is inherent in holography, as opposed to currently available optical devices such as CDs or DVDs based on surface recording. For this reason, the theoretical information storage capacity of holographic memories greatly exceeds the capacity of current techniques: It is 1000 times greater than a CDROM, with a random access time of only $10 \%$ of the latter.

One of the basic requirements for holographic memories to be competitive is that the thickness of the recording material layer must be $500 \mu \mathrm{m}$ or more. ${ }^{1}$ A greater number of holograms may be re-

All the authors are with the Universidad de Alicante, Apartado 99, E-03080 Alicante, Spain. S. Gallego (sergi.gallego@ua.es), M. Ortuño, C. Neipp, A. Márquez, and A. Beléndez are with the Departamento de Física, Ingeniería de Sistemas y Teoría de la Señal. I. Pascual is with the Departamento Interuniversitario de Óptica.

Received 1 February 2005; revised manuscript received 18 May 2005; accepted 19 May 2005.

0003-6935/05/296205-06\$15.00/0

(C) 2005 Optical Society of America corded with thicker layers, since in this case the angular Bragg selectivity is higher and the width of the angular response curve is very small. ${ }^{3}$ It is not easy to find such a great thickness with the recording materials currently available, in spite of the large number of materials that have been employed as holographic memories (e.g., photorefractive polymers, bacteriorhodopsin, photopolymers). ${ }^{2-6}$ Photopolymers based on an acrylamide (AA) monomer are one of the materials of interest because of their good properties, in particular their acceptable energetic sensitivity compared with that of other available materials, the possibility of easily adapting their spectral sensitivity to the type of recording laser used by simply changing the sensitizer dye, and high diffraction efficiency, together with an acceptable resolution and signal/ noise ratio. ${ }^{7}$ Specifically, our research team uses polyvinyl alcohol/acrylamide (PVA/AA) compounds. ${ }^{8,9}$ Their low price, easy preparation, and the fact that complicated developing processes are not necessary make them even more attractive for use on a large scale in read-only write-once-read-many type memories.

Photopolymers have many attractive applications in addition to holographic data storage, for example, optical imaging processing by Bragg diffraction ${ }^{10}$ and generation of diffraction elements. ${ }^{11}$ For this reason many models have been proposed to simulate the behavior of this type of material. ${ }^{12-15}$ The usefulness 
of these models has been demonstrated in different studies (to predict the cutoff spatial frequencies, the residual monomer, etc.), ${ }^{16,17}$ but the thickness of the layers analyzed by the models is only around $100 \mu \mathrm{m}$. In this paper the main idea is to study the behavior of the holographic memories recorded in PVA/AA (layers with an effective optical thickness higher than $500 \mu \mathrm{m}$ ) using a diffusion model. The first goal of our study is to estimate the parameters (diffusivity, polymerization rate, etc.) that characterize the hologram formation in the material. The values obtained are compared with the parameters obtained in gratings with a thickness of $100 \mu \mathrm{m}$. The second goal is to compare the polymer diffusivity in thin and thick layers. For this analysis we use a method based on a model proposed by O'Neill et al. ${ }^{18}$ These values are important to determine the properties of the photopolymer (chain length, viscosity, etc.). Cross-linker monomers are introduced in the composition to conserve the information stored in the photopolymers and to stop polymer diffusion. ${ }^{19}$ Nevertheless, the values of material viscosity are similar in the two cases because the cross-linker concentration is very low. ${ }^{19,20}$

\section{Theoretical Model}

To analyze the photopolymer characteristics we will use the diffusion model described in Ref. 15. This is a first-harmonic diffusion model; thus higher harmonics are not considered in the Fourier expansion of the monomer and polymer concentrations. This approximation can be justified because the higher harmonics of the refractive index are negligible in gratings recorded in PVA/AA, as demonstrated in Ref. 21. In a first-harmonic diffusion model a sinus (or cosinus) distribution is considered for the refractive index $(n)$, monomer concentration $([M])$, and polymer concentration $([P])$ :

$$
\begin{gathered}
n(x, t)=n_{0}(t)-n_{1}(t) \cos \left(K_{g} x\right), \\
{[M](x, t)=[M]_{0}(t)-[M]_{1}(t) \cos \left(K_{g} x\right),} \\
{[P](x, t)=[P]_{0}(t)+[P]_{1}(t) \cos \left(K_{g} x\right),}
\end{gathered}
$$

where $K_{g}$ is the grating period and the refractiveindex modulation $n_{1}(t)$ depends on the refractive indices of the different components of the material as in Ref. 22:

$$
\begin{aligned}
n_{1}= & \frac{\left(n_{\text {dark }}^{2}+2\right)^{2}}{6 n_{\text {dark }}}\left[-\left(\frac{n_{m}^{2}-1}{n_{m}^{2}+2}-\frac{n_{b}^{2}-1}{n_{b}^{2}+2}\right)[M]_{1}\right. \\
& \left.+\left(\frac{n_{p}{ }^{2}-1}{n_{p}{ }^{2}+2}-\frac{n_{b}{ }^{2}-1}{n_{b}{ }^{2}+2}\right)[P]_{1}\right],
\end{aligned}
$$

where $n_{p}$ is the polymer refractive index, $n_{m}$ is the monomer refractive index, $n_{b}$ is the binder refractive index, and $n_{\text {dark }}$ is the refractive index of the layer before exposure. In the material used in this work the different refractive indices take the following values: $n_{m}=1.486, n_{b}=1.474$, and $n_{\text {dark }}=1.478$. These values were obtained using the Lorentz-Lorenz equation and the method used is described in Ref. 22 . The calculations are based on refractometer measurements by use of water solutions.

If a sinusoidal interference pattern is considered, the polymerization $(F)$ can be obtained as

$$
F(x, t)=k_{R}(t) I_{0}^{\gamma}\left[1+V \cos \left(K_{g} x\right)\right]^{\gamma}=k_{R}(t) I^{\gamma},
$$

where $V$ is the visibility of the fringes, $I$ is the intensity, $\gamma$ indicates the relationship between the intensity and the polymerization, and $k_{R}$ is the polymerization rate. The polymerization rate decreases because of the Trommsdorff effect and is given by

$$
k_{R}(t)=k_{R} \exp \left(-\varphi I_{0} t\right),
$$

where $\varphi$ is the attenuation coefficient of the polymerization rate.

The differential equations to be solved are

$$
\begin{aligned}
& \frac{\partial[M](x, t)}{\partial t}=\frac{\partial}{\partial x} D \frac{\partial[M](x, t)}{\partial x}-k_{R}(t) I^{\gamma}(x)[M](x, t), \\
& \frac{\partial[P](x, t)}{\partial t}=k_{R}(t) I^{\gamma}(x)[M](x, t),
\end{aligned}
$$

where $D$ is the monomer diffusion constant. In this paper a value of 0.5 for $\gamma$ is considered. ${ }^{12}$

Limitation of the diffusivity due to the increase in polymer concentration is also considered, as can be seen in the following equation:

$$
D(t)=D \exp \left(-\varphi^{\prime} I_{0} t\right)
$$

where $\varphi^{\prime}$ is the attenuation coefficient of the polymerization rate and is similar to $\varphi$.

The physical thickness of the material $d^{\prime}$ is larger than the effective optical thickness $(d)$ occupied by the recorded grating. We call $d$ the effective optical thickness of the material. This difference is due to the existence of an exponential decay of the light across the depth of the hologram. An algorithm based on the rigorous coupled-wave method $^{21}$ was developed to study this decay and obtain the effective optical thickness of the holographic memories ${ }^{23}$ as well as the thickness where the grating is recorded. This parameter is then introduced in the diffusion model.

\section{Experimental Setup}

The photopolymerizable solution was prepared by adding yellowish eosin (the dye), together with a solution of AA (the monomer) and triethanolamine (TEA) (the coinitiator), to a polyvinyl alcohol (PVA) (the binder) solution. In this study the solutions were prepared using a conventional magnetic stirrer, under red light and in standard laboratory conditions (temperature, pressure, relative humidity). If the vis- 
Table 1. Composition of the Solution Used to Prepare Layers of the Recording Material According to the Thickness to be Obtained

\begin{tabular}{lcc}
\hline \multicolumn{1}{c}{ Component } & More than $500 \mu \mathrm{m}$ & Around $100 \mu \mathrm{m}$ \\
\hline PVA $M_{w}=130,000$ & $13.30 \% \mathrm{w} / \mathrm{v}^{a}$ & $8.5 \% \mathrm{w} / \mathrm{v}$ \\
TEA & $0.15 \mathrm{M}$ & $0.4 \mathrm{M}$ \\
AA & $0.34 \mathrm{M}$ & $0.48 \mathrm{M}$ \\
Yellowish eosin & $9.00 \times 10^{-5} \mathrm{M}$ & $2.4 \times 10^{-4} \mathrm{M}$ \\
\hline
\end{tabular}

${ }^{a} \mathrm{w} / \mathrm{v}$, weight by volume.

cosity is too high to use a magnetic stirrer, we stir the solution slowly by hand to prevent the formation of air bubbles and then apply a vacuum atmosphere to both the prepared solution and the coated molds so that any eventual air bubbles are completely eliminated. The solutions are deposited by gravity in polystyrene circular molds and left in the dark to allow the water to evaporate while recording under laboratory conditions (temperature and relative humidity) during the process. When a high percentage of the water content has evaporated, the dry material is removed from the mold, cut into squares, and deposited, without the need of adhesive, onto the surface of glass plates measuring $6.5 \mathrm{~cm} \times 6.5 \mathrm{~cm}$. The plates are then ready for exposure, which takes place immediately. Table 1 gives both the composition of the solution of the holographic memories (more than 500 $\mu \mathrm{m}$ thick) and the $100 \mu \mathrm{m}$ thick layers. To introduce the monomer concentration in the dry film, the amount of water evaporated is calculated. ${ }^{8}$ The values (in volume fraction units) of the initial monomer concentration in the dry holographic memories used in this work are 0.19 and 0.25 for thick and thin layers, respectively. These are the values to be introduced in the model.

To study the behavior of the photopolymeric layers, we obtained unslanted diffraction gratings using a holographic setup (Fig. 1). An argon laser at a wavelength of $514 \mathrm{~nm}$ was used to store diffraction gratings by means of continuous laser exposure. The laser beam was split into two secondary beams with an intensity ratio of $1: 1$. The diameters of these beams were increased to $1.5 \mathrm{~cm}$ with an expander, while spatial filtering was ensured. The object and reference beams were recombined at the sample at an

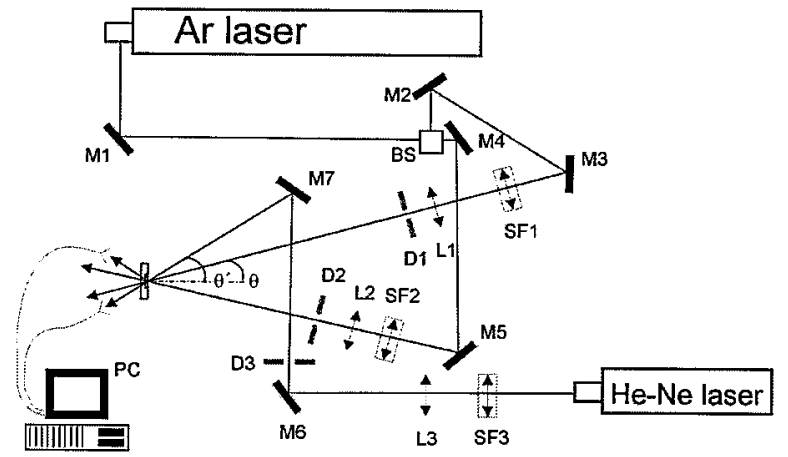

Fig. 1. Experimental setup. Mi, mirrors; Li, lenses; SF $i$, spatial filters to expand the beams; $i$, diaphragms; BS, beam splitter.

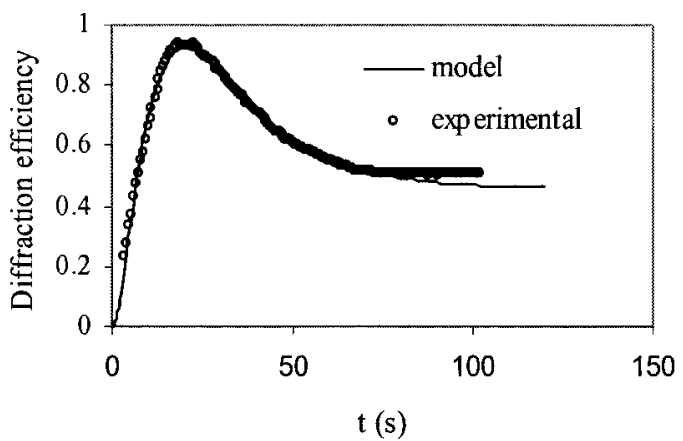

Fig. 2. Diffraction efficiency versus exposure time at a Bragg angle for a thin grating (112 $\mu \mathrm{m}$ thick).

angle of $16.8^{\circ}$ to the normal with an appropriate set of mirrors, and the spatial frequency obtained was 1125 lines $/ \mathrm{mm}$. The working intensity at $514 \mathrm{~nm}$ was $5 \mathrm{~mW} / \mathrm{cm}^{2}$. The diffracted and transmitted intensity were monitored in real time with a $\mathrm{He}-\mathrm{Ne}$ laser positioned at Bragg's angle $\left(20.8^{\circ}\right)$ tuned to $633 \mathrm{~nm}$, where the material is not sensitive. To obtain the transmission and diffraction efficiency as a function of the angle at reconstruction, we placed the plates on a rotating stage. The transmission and diffraction efficiency were calculated as the ratio of the transmitted and diffracted beam power, respectively, to the incident power. We also consider the losses due to the Fresnel reflection coefficients.

\section{Results and Discussion}

\section{A. Hologram Formation}

In this section we use the diffusion model proposed in Ref. 15 to characterize the holographic behavior of layers with a physical thickness greater than $500 \mu \mathrm{m}$. To obtain a deeper insight, first we analyze the characteristics of thin layers, then we compare these values with the results provided by thick layers.

\section{Thin Layers}

Figure 2 shows the diffraction efficiency at the Bragg angle of incidence as a function of the exposure time for a transmission grating recorded on a thin layer of photopolymer (112 $\mu \mathrm{m}$ thick). The values are obtained using a monitoring beam of the $\mathrm{He}-\mathrm{Ne}$ laser. The experimental data are represented as open circles, whereas the theoretical curve obtained using the model in Ref. 15 is represented as a continuous curve. Good agreement between the experimental data and the model can be seen. It is interesting to note that at Bragg's angle for an exposure time longer than $60 \mathrm{~s}$ the value of the diffraction efficiency does not change. This phenomenon is due to the saturation of the photopolymer since the free monomer in the bright regions has been consumed. Nevertheless, there is a residual monomer in the dark regions, as can be seen in Ref. 24: The value of the residual monomer concentration in dark regions is around $30 \%$ of that of the initial monomer. 


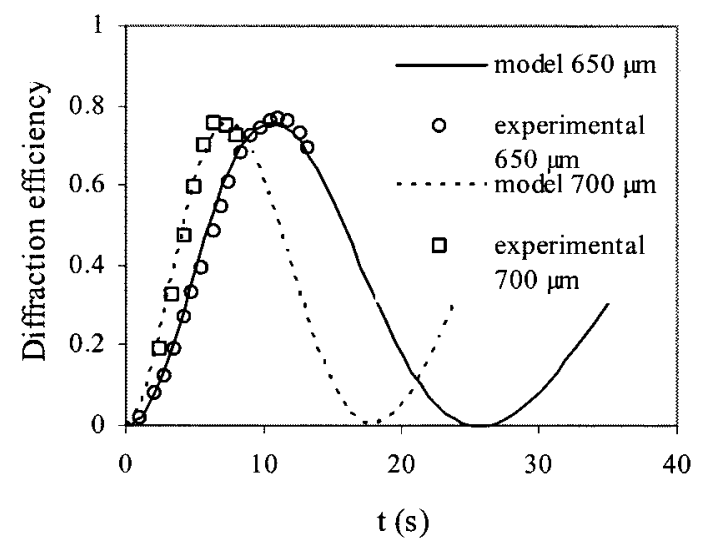

Fig. 3. Diffraction efficiency versus the exposure time at a Bragg angle for thick layers with two different thickness.

The specific values for the parameters obtained with the model are $D=2 \times 10^{-10} \mathrm{~cm}^{2} / \mathrm{s}, k_{R}=$ $0.025 \mathrm{~cm} \mathrm{~mW}^{-1 / 2} \mathrm{~s}^{-1}, n_{p}=1.504$, and $\varphi=0.005$ $\mathrm{cm}^{2} \mathrm{~mW}^{-1} \mu \mathrm{m}^{-1}$.

It is interesting to note the high value of diffusivity obtained, as compared with that found in previous studies ${ }^{17}$ which is due to a very high concentration of TEA. This component is liquid at ambient temperature and facilitates the diffusion of the species inside the material. ${ }^{25}$

\section{Thick Layers}

Figure 3 shows diffraction efficiency as a function of exposure time for two gratings recorded in holographic memories (layers thicker than $500 \mu \mathrm{m}$ ). The gratings studied have a different effective optical thickness: The first (open circles) is $650 \mu \mathrm{m}$ thick and the second (open squares) is $700 \mu \mathrm{m}$ thick, as can be seen in Fig. 3. The model correctly predicts the behavior in both cases. After the first diffraction efficiency maximum is reached, the effects of overmodulation of the refractive index can be observed. ${ }^{25}$ Exposure was stopped near this maximum because scattering increases dramatically for high exposures as does attenuation of the index profile across the depth of the layer. 8 The combination of these two factors produces a high distortion of the angular response. As a result we cannot properly fit the value of the effective optical thickness, which is needed in the simulations of diffraction efficiency.

The parameters obtained using the first-harmonic diffusion model for the holographic memories are $D$ $=7 \times 10^{-10} \mathrm{~cm}^{2} / \mathrm{s}, k_{R}=0.016 \mathrm{~cm} \mathrm{~mW}^{-1 / 2} \mathrm{~s}^{-1}, n_{p}=$ 1.504, and $\varphi=0.005 \mathrm{~cm}^{2} \mathrm{~mW}^{-1} \mu \mathrm{m}^{-1}$.

As can be seen, the values of the parameters are similar to those obtained when thin layers were studied, although there are some slight changes because the composition and the thickness are different. In the first place thick layers present higher diffusivity than thin layers, and in the second place the initial polymerization rate is also low. This low polymerization rate is due to low concentrations of TEA, so the light absorption decreases with the result that we obtain high values of effective optical thickness. ${ }^{8,9} \mathrm{On}$

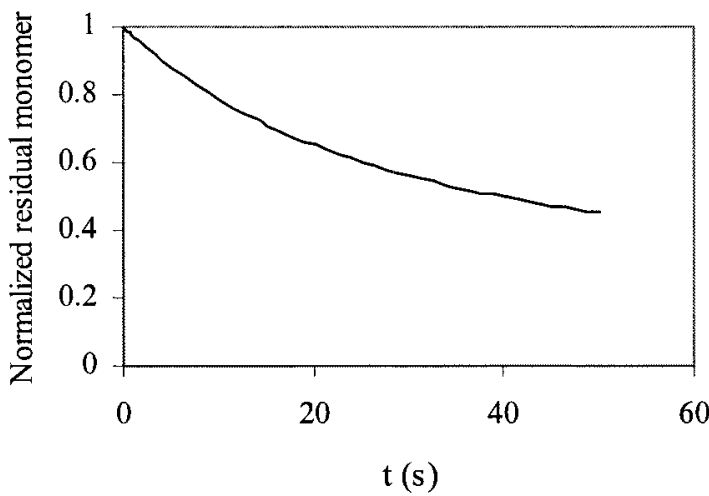

Fig. 4. Normalized residual free monomer $\left[\mathrm{M}_{0}\right]$ [see Eq. (2)] as a function of the exposure time.

the other hand, the concentration of PVA is high in holographic memories, and this produces stronger layers but a low polymerization rate. ${ }^{8}$

Let us analyze the stability of recorded gratings. Low polymerization rates and short exposure times lead to higher values of residual monomer, and this can be considered a drawback to the conservation of information stored in this type of holographic memory. The evolution of the free monomer $\left[\mathrm{M}_{0}\right]$ [see Eq. (2)] as a function of time can be analyzed in Fig. 4. In Fig. 4, after $20 \mathrm{~s}$ (the optimum exposure time for this type of grating) the concentration of the residual monomer is around $60 \%$. This high value is an important drawback for the stability of the information stored: Under white light the monomers will react.

Another important difficulty associated with these layers of great physical thickness is the accurate determination of the effective optical thickness: The reason is the existence of an attenuation of the index profile in depth. ${ }^{24}$ The initial transmittance (for a wavelength of $514 \mathrm{~nm}$ ) of this type of layer is around $1 \%$, and the main portion of the information is stored in the vicinity of the entrance side of the layer. This prevents narrow angular responses from being obtained. The angular response fitted by the algorithm proposed in Ref. 24 for a grating of $650 \mu \mathrm{m}$ is plotted in Fig. 5.

The parameters obtained fitting the angular response are effective optical thickness $d=650 \mu \mathrm{m}$, average index modulation $n_{1}=0.00035$, and exponential decay of the index modulation $\alpha=$ $0.005 \mu \mathrm{m}^{-1}$. We consider the following index decay:

$$
n_{1}(z)=n_{1} \exp (-\alpha z) .
$$

\section{B. Polymer Diffusion}

In PVA/AA-based photopolymers (without crosslinker monomers in their composition), estimation of the polymer diffusion $D$ is a simple task when we use the model proposed by O'Neill et al. ${ }^{18}$ The value of this parameter indicates the viscosity of the material and provides useful information as to the general behavior of the photopolymer during hologram formation and also when the hologram has been stored. If cross-linker monomers are used, the monomer chains are very 


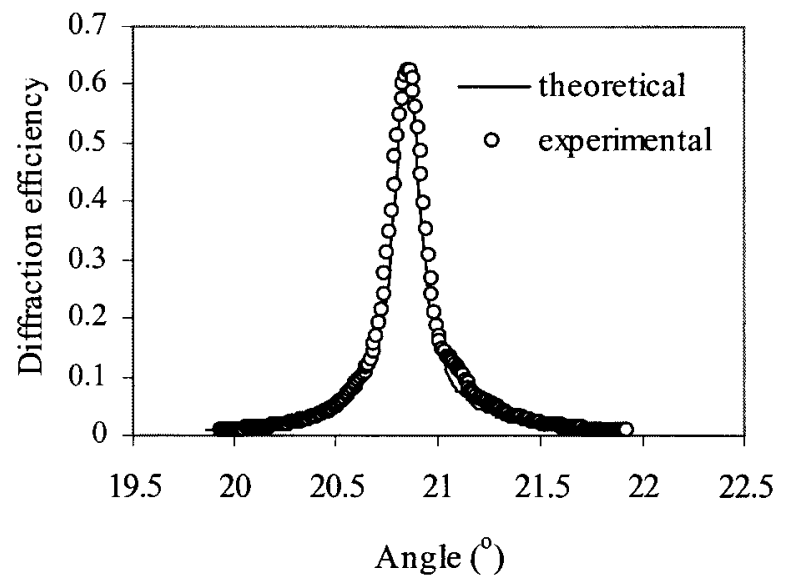

Fig. 5. Angular scan around the first Bragg angle is plotted for holographic memories (650 $\mu \mathrm{m}$ of thickness).

large, the diffusion is very slow, and this effect cannot be seen. The values of polymer diffusion depend basically on the same factors as the monomer diffusion photopolymer composition (PVA, monomer, etc.), humidity, temperature, and drying time. The method used to calculate the polymer diffusivity inside the material is simple. In the model an exponential decrease in index modulation after recording the hologram is assumed using a constant value of diffusivity. This idea can be expressed in the following form ${ }^{18}$ :

$$
n_{1}(t)=n_{1}(t \rightarrow \infty)+\Delta n_{1} \exp \left[-D\left(\frac{2 \pi}{\Lambda}\right)^{2} t\right]
$$

We measured the diffraction efficiency decay in the recorded gratings, and from the fitting of the angular responses we found the values of $n_{1}$.

\section{Thin Layers}

First let us analyze the results for the thin layers ( $\sim 100 \mu \mathrm{m}$ thick). In the particular composition used in this work the diffraction efficiency after $500 \mathrm{~min}$ is very low. Using Eq. (11), Fig. 6 can be obtained. From the fitting of the angular responses we find that the

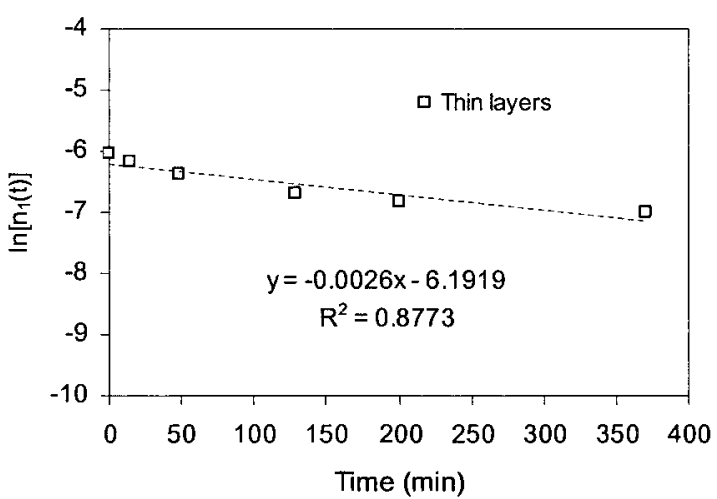

Fig. 6. Natural logarithm of the exponential decay of $n_{1}$, modulation of the refractive index, as a function of time plotted for thin layers. The correlation value for the line fit is also presented.

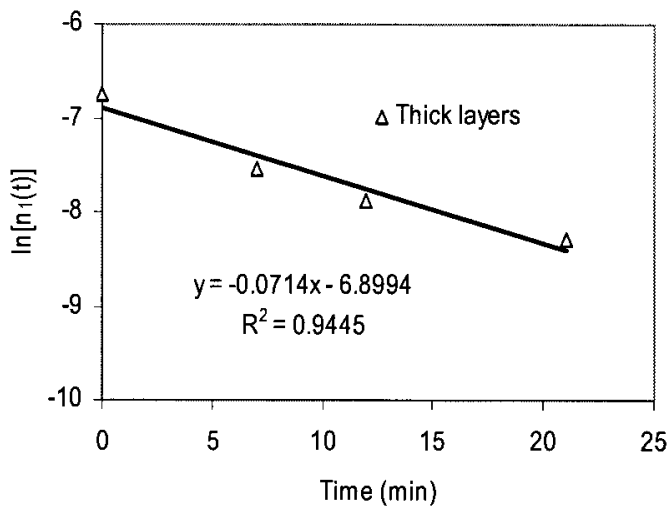

Fig. 7. Natural logarithm of the exponential decay of $n_{1}$, modulation of the refractive index, as a function of the time plotted for thick layers. The correlation value for the line fit is also presented.

polymer diffusivity is around $8.7 \times 10^{-15} \mathrm{~cm}^{2} / \mathrm{s}$, which is smaller than the value obtained by O'Neill et al. for photopolymer films with similar thickness $\left(D \sim 3 \times 10^{-13} \mathrm{~cm}^{2} / \mathrm{s}\right)$. The value of $R^{2}$ indicates the correlation and the quality of the fit. This value is not optimal because the drying process of the layer has not finished, ${ }^{8}$ thus $D$ is still not stabilized.

\section{Thick Layers}

When the same study is applied to thick gratings, the value obtained for the polymer diffusion in thick layers is around $1.6 \times 10^{-14} \mathrm{~cm}^{2} / \mathrm{s}$. The value of $R^{2}$ is similar to that obtained in the case of thin layers. This value of polymer diffusivity is higher than the one for thin layers but lower than the value obtained by O'Neill et al. An interesting situation occurs when, in the thick layer composition, a new component (liquid in laboratory conditions) is introduced. The function of this liquid is to increase the diffusivity of the material. In the experiment described in this paper, the liquid used is glycerine because it does not react with the other substances. In Fig. 7 the behavior of the grating after exposure can be observed. The estimated value of polymer chain diffusivity is around $1.4 \times 10^{-13}$ $\mathrm{cm}^{2} / \mathrm{s}$. For this type of grating the diffraction efficiency is already very low $25 \mathrm{~min}$ after the recording process. Analyzing these results, it is clear that the diffusivity for the different substances is higher when a liquid is introduced in the polymer composition. In this case the value of the correlation coefficient is close to 1 , which indicates the quality of the fit.

This diffusivity analysis is important to determine the stability of the recorded holograms in dark conditions. ${ }^{18,24}$ In general, the addition of a cross-linking monomer will stabilize the hologram. However, in high fluid photopolymer systems the high diffraction efficiencies achieved during recording may be lost later.

\section{Conclusions}

The validity of the first-harmonic diffusion model to predict the behavior of gratings stored in holographic memories based on PVA/AA photopolymers is demonstrated. A first estimation of parameters that gov- 
ern the formation of the hologram in photopolymers (monomer diffusivity and polymerization rate) has been done. These values are compared with the values for thin layers (around $100 \mu \mathrm{m}$ ). The polymerization rates in holographic memories are low, and this results in higher values of residual monomer concentration. The values of monomer diffusion are higher for thick layers.

As in the cases studied, cross-linker monomers are not included in the material composition, and the polymer diffusion can be observed and measured. Holographic memories present a higher polymer diffusion than thin layers. Nevertheless, in the references similar materials with higher values of polymer diffusion have been described. The diffusion of the different species can be increased using an inert liquid in the polymer composition to better observe the polymer diffusion, thereby generating more accurate information about the behavior of thick photopolymer materials. To obtain more accurate information about the behavior of holographic memories based on PVA/AA photopolymers during the recording process, it is necessary to employ models that take into account the attenuation of the intensity in depth.

This work was supported by the Dirección General de Investigación y Transferencia Tecnológica (Generalitat Valenciana, Spain) under projects GV04A/565 and GV04A/574.

\section{References}

1. H. J. Coufal, D. Psaltis, and G. T. Sincerbox, eds., Holographic Data Storage (Springer-Verlag, 2000).

2. J. E. Boyd, T. J. Trentler, K. W. Rajeev, Y. I. Vega-Cantu, and V. L. Colvin, "Effect of film thickness on the performance of photopolymers as holographic recording materials," Appl. Opt. 39, 2353-2358 (2000).

3. P. Cheben and M. L. Calvo, "A photopolymerizable glass with diffraction efficiency near 100\% for holographic storage," Appl. Phys. Lett. 78, 1490-1492 (2001).

4. S. S. Orlov, W. Philips, E. Bjormson, Y. Takashima, P. Sundaram, L. Hesselink, R. Okas, D. Kwan, and R. Snyder, "High-transfer-rate high-capacity holographic disk datastorage system," Appl. Opt. 43, 4902-4914 (2004).

5. D. A. Waldman, C. J. Butler, and D. H Raguin, "CROP holographic storage media for optical data storage at greater than $100 \mathrm{bits} / \mu \mathrm{m}^{2}$, in Organic Holographic Materials and Applications, K. Meerholz, ed., Proc. SPIE 5316, 10-25 (2003).

6. A. S. Bablumian and T. F. Krile, "Multiplexed holograms in thick bacteriorhodopsin films for optical memory/interconnections," Opt. Eng. 39, 2964-2974 (2000).

7. S. Blaya, L. Carretero, and A. Fimia, "Highly sensitive photopolymerisable dry film for use in real time holography," Appl. Phys. Lett. 75, 1628-1630 (1998).

8. M. Ortuño, S. Gallego, C. García, C. Neipp, A. Beléndez, and I. Pascual, "Optimization of a $1 \mathrm{~mm}$ thick PVA/acrylamide recording material to obtain holographic memories: method of preparation and holographic properties," Appl. Phys. B 76, 851-857 (2003).

9. M. Ortuño, S. Gallego, C. García, C. Neipp, and I. Pascual, "Holo- graphic characteristics of a 1-mm-thick photopolymer to be used in holographic memories," Appl. Opt. 42, 7008-7012 (2003).

10. A. Márquez, C. Neipp, A. Beléndez, S. Gallego, M. Ortuño, and I. Pascual, "Edge-enhanced imaging with polyvinyl alcohol/acrylamide photopolymer gratings," Opt. Lett. 28, 1510-1512 (2003).

11. W. Gambogi, K. Steijn, S. Mackara, T. Duzik, B. Hamzavy, and J. Kelly, "HOE imaging in DuPont holographic photopolymers," in Diffractive and Holographic Optics Technology, I. Cindrich and S. H. Lee, eds., Proc. SPIE 2152, 282-293 (1994).

12. G. Zhao and P. Mouroulis, "Diffusion model of hologram formation in dry photopolymer materials," J. Mod. Opt. 41, 19291939 (1994).

13. J. H. Kwon, H. C. Hwang, and K. C. Woo, "Analysis of temporal behavior of beams diffracted by volume gratings formed in photopolymers," J. Opt. Soc. Am. B 16, 1651-1657 (1999).

14. J. T. Sheridan and J. R. Lawrence, "Nonlocal-response diffusion model of holographic recording in photopolymer," J. Opt. Soc. Am. A 17, 1008-1014 (2000).

15. C. Neipp, S. Gallego, M. Ortuño, A. Márquez, M. Álvarez, A. Beléndez, and I. Pascual, "First-harmonic diffusion-based model applied to a polyvinyl alcohol-acrylamide-based photopolymer," J. Opt. Soc. Am. B 20, 2052-2060 (2003).

16. J. R. Lawrence, F. T. O'Neill, and J. T. Sheridan, "Photopolymer holographic recording material parameter estimation using a non-local diffusion based model," J. Appl. Phys. 90, 3142-3148 (2001).

17. S. Gallego, C. Neipp, M. Ortuño, A. Beléndez, and I. Pascual, "Stabilization of volume gratings recorded in PVA/acrylamide photopolymers with diffraction efficiencies higher than 90\%," J. Mod. Opt. 51, 491-503 (2004).

18. F. T. O'Neill, J. R. Lawrence, and J. T. Sheridan, "Improvement of holographic recording material using aerosol sealant," J. Opt. 3, 20-25 (2001).

19. S. Martin, C. A. Feely, and V. Toal, "Holographic recording characteristics of an acrylamide-based photopolymer," Appl. Opt. 36, 5757-5768 (1997).

20. C. Neipp, S. Gallego, M. Ortuño, A. Márquez, A. Beléndez, and I. Pascual, "Characterization of a PVA/acrylamide photopolymer. Influence of a cross-linking monomer in the final characteristics of the hologram," Opt. Commun. 224, 27-34 (2003).

21. C. Neipp, A. Beléndez, S. Gallego, M. Ortuño, I. Pascual, and J. T. Sheridan, "Angular responses of the first and second diffracted orders in transmission diffraction grating recorded on photopolymer material," Opt. Express 11, 1835-1843 (2003).

22. I. Aubrecht, M. Miler, and I. Koudela, "Recording of holographic diffraction gratings in photopolymers: theoretical modelling and real-time monitoring of grating growth," J. Mod. Opt. 45, 1465-1477 (1998).

23. C. Neipp, J. T. Sheridan, S. Gallego, M. Ortuño, A. Márquez, I. Pascual, and A. Beléndez, "Effect of a depth attenuated refractive index profile in the angular responses of the efficiency of higher orders in volume gratings recorded in a PVA/acrylamide photopolymer," Opt. Commun. 233, 311-322 (2004).

24. S. Gallego, C. Neipp, M. Ortuño, A. Márquez, A. Beléndez, and I. Pascual, "Diffusion-based model to predict the conservation of holographic gratings recorded in polyvinyl(alcohol)acrylamide photopolymer," Appl. Opt. 42, 5839-5845 (2003).

25. S. Gallego, M. Ortuño, C. Neipp, C. Garcia, A. Beléndez, and I. Pascual, "Temporal evolution of the angular response of a holographic diffraction grating in PVA/acrylamide photopolymer," Opt. Express 11, 181-190 (2003). 